\title{
Physicochemical Characteristics of the Okomu Wetland, Edo State, Nigeria
}

\author{
Ogbeibu A. E. ${ }^{1, *}$ and Ehiorobo S. I. ${ }^{2}$ \\ ${ }^{1,2}$ Department of Animal and Environmental Biology, Faculty of Life Sciences, University of Benin, Benin City, \\ Nigeria \\ Corresponding Author: *ogbeibu@yahoo.com
}

https://doi.org/10.36263/nijest.2020.02.0225

\begin{abstract}
Water quality assessment was carried out within the $202 \mathrm{~km}^{2}$ Okomu National Park located within the 1,082 $\mathrm{km}^{2}$ Okomu Forest Reserve between January 2017 and December 2018. Water sampling was carried in ten compartments of approximately $1.6 \mathrm{~km}^{2}$ each, covering Ponds, Stream and River, based on easy accessibility. No such study has been carried out for the Okomu Wetlands in the past three decades. Sampling protocols, laboratory analysis and quality control/quality assurance measures followed standard procedures. All physicochemical parameters showed significant $(P<$ 0.001) spatial variations. The maximum and minimum values obtained for physicochemical parameters of the Okomu Wetland are 23.6 and $38.0^{\circ} \mathrm{C}$ air temperature; $21.0-38^{\circ} \mathrm{C}$ water temperature; 0.15 - $1.02 \mathrm{~m}$ water level; 16.7 - 150.7mg/l total dissolved Solids (TDS); 0.5 $18.8 \mathrm{mg} / \mathrm{l}$ total suspended Solids (TSS); 0.2 - $14.8 \mathrm{mg} / \mathrm{l}$ turbidity; $40.0-307.4 \mu \mathrm{s} / \mathrm{cm}$ electrical conductivity (EC); 4.4 - $6.7 \mathrm{pH} ; 0.02$ - 0.14mg/l salinity; 0.7 - 5.5mg/l dissolve oxygen (DO); 0.5 $3.8 \mathrm{mg} / \mathrm{l}$ biochemical oxygen demand (BOD); 1.5 - $120.2 \mathrm{mg} / \mathrm{l}$ chemical oxygen demand (COD); 23.8 - 593.6mg/l bicarbonates $\left(\mathrm{HCO}_{3}\right) ; 69.9$ - 245.3mg/l Chlorine $(\mathrm{Cl}) ; 0.02$ - 0.59mg/l nitrite $\left(\mathrm{NO}_{2}\right) ; 0.11$ - 2.34mg/l nitrate $\left(\mathrm{NO}_{3}\right) ; 0.04-2.11 \mathrm{mg} / \mathrm{l}$ ammonium-nitrogen $\left(\mathrm{NH}_{4} \mathrm{~N}\right) ; 0.05-2.96 \mathrm{mg} / \mathrm{l}$ sulphate (SO $\left.\mathrm{SO}_{4}\right) ; 0.09$ - 9.2mg/l phosphorus $(\mathrm{P}) ; 0.20$ - 2.72mg/l sodium $(\mathrm{Na}), 0.03-1.32 \mathrm{mg} / \mathrm{l}$ potassium $(\mathrm{K}) ; 0.88$ - $5.88 \mathrm{mg} / \mathrm{l}$ calcium $(\mathrm{Ca}) ; 0.13-3.1 \mathrm{mg} / \mathrm{l}$ magnesium $(\mathrm{Mg}) ; 5.8-18.9 \mathrm{mg} / \mathrm{l}$ iron $(\mathrm{Fe}) ; 0.04$ - 1.1mg/l manganese $(\mathrm{Mn}) ; 0.02$ - 0.09mg/l copper $(\mathrm{Cu}) ; 0.93$ - $6.0 \mathrm{mg} / \mathrm{l}$ zinc $(\mathrm{Zn}) ; 0.01$ $2.9 \mathrm{mg} / \mathrm{l} \mathrm{lead}(\mathrm{Pb}) ; 0.01-0.18 \mathrm{mg} / \mathrm{l} \mathrm{cadmium}(\mathrm{Cd}) ; 0.06$ - 4.0mg/l chromium $(\mathrm{Cr}) ; 0.01-0.15 \mathrm{mg} / \mathrm{l}$ nickel (Ni); 0.01 - $11 \mathrm{mg} / \mathrm{l}$ vanadium $(\mathrm{V})$. Air and water temperatures were mostly influenced by forest canopy cover. $\mathrm{pH}$ levels indicate that the water bodies within the forested wetland are slightly acidic. Higher DO values were recorded in the Arhakhuan Stream and Okomu River (Agekpukpu and Iron bridge) than in the temporary ponds. BOD levels of greater than $1 \mathrm{mg} / \mathrm{l}$ were observed which is indicative of slight levels of organic pollution. Higher concentrations of TDS, TSS, turbidity, EC, Colour, $\mathrm{HCO}_{3}, \mathrm{NH}_{4} \mathrm{~N}, \mathrm{NO}_{2}, \mathrm{NO}_{3}, \mathrm{P}, \mathrm{Na}, \mathrm{K}, \mathrm{Ca} \mathrm{Mg}$ and heavy metals were recorded in the temporary ponds than in the Stream and River. Research on water bodies within wetlands should be carried out routinely in order to monitor changes in the water conditions that could occur overtime whether natural or anthropogenic. This becomes even more pertinent in the light of glaring impacts of climate change and increasing environmental modifications.
\end{abstract}

Keywords: Wetland, Okomu National Park, Physicochemical parameters, Pond, Stream, River, Water quality

\subsection{Introduction}

Wetlands include "areas of marsh, fen, peat-land or water, whether natural or artificial, permanent or temporary, with water that is static or flowing, fresh, brackish or salt, including areas of marine water, the depth of which at low tide does not exceed 6m" (Ramsar Convention Secretariat, 2007). Wetlands are the only ecosystems that have their own international convention (Turner et al., 2000) and they also gave rise to the first modern global nature conservation convention (Matthews 1993). Uluocha and Okeke (2004) estimated that $28,000 \mathrm{~km}^{2}$ (about $3 \%$ ) of the $923,768 \mathrm{~km}^{2}$ land surface in Nigeria is covered with wetlands. They are among the most important ecosystems in the world and perform essential ecosystem services including preservation of biodiversity and providing habitat for many 
endangered species amongst others (Barbier et al., 1997, Asibor, 2009). The enormous benefits and values of wetlands have attracted increasing worldwide importance in agriculture, aquaculture and environmental conservation (Okonkwo et al., 2015); therefore, the need to protect the remaining wetlands (Millennium Ecosystem Assessment, 2005). In Nigeria, seven (7) protected areas have been identified including the Okomu National Park (Olaleru and Egonmwan, 2014) which covers an area of $202 \mathrm{~km}^{2}$ (about 15\%) of the $1,082 \mathrm{~km}^{2}$ covered by the then Okomu Forest Reserve (Emelue and Ukandu, 2014). In the Okomu Forest Reserve abound many temporary and permanent water bodies (Ogbeibu and Victor, 1995). The physicochemical parameters of water can be investigated to assess the ecological nature of the wetlands (Bamgboye et al., 2016).

Despite the conservative status of the Okomu National Park where the study was conducted, there is need to routinely monitor and document the levels of concentrations of physicochemical parameters of water bodies within the wetland in order to identify possible sources of pollution whether natural or anthropogenic and appropriate actions taken where and when necessary. The present study updates the physicochemical status of the wetland which has not received any attention in recent times. The last related work on physicochemical characteristics carried out in the study area is those of Ogbeibu and Victor (1995). This study is unique in that, it covers different types of freshwater bodies (Pond, Stream and River) which is not a very common trend in research especially this part of the world. As part of trend analysis and routine environmental monitoring studies, this paper documents the current status of the physicochemical parameters of water bodies within the Okomu Wetland.

\subsection{Methodology}

\subsection{Study area}

The Okomu Forest Reserve is in the lowland tropical rainforest $\left(6.5^{\circ} \mathrm{N} ; 5.8^{\circ} \mathrm{E}\right)$, about $75 \mathrm{~km}$ West of Benin City, situated between the Rivers Osse and Siluko, Edo State, Nigeria (Ogbeibu and Egborge, 1995; Ajayi, 2001). The Okomu National Park which was formerly known as the Okomu Forest Reserve is located in the Southern Part of Nigeria and was rated the largest and least degraded of all such Reserves in the country (Anadu and Oates, 1982). It is characterized with distinct humid rainy and dry season (Ogbeibu and Victor, 1995). Detailed descriptions were given by Jones (1955), Ogbeibu and Egborge (1995), Nigeria National Park Service (2015). A total of ten sampling stations from ten compartments within the Okomu National Park were designated for the study and physicochemical parameters sampled monthly. Sampling stations were selected based on easy accessibility. The sampling spanned 2 years, covering the rainy and dry months. The Okomu National Park is compartmented and each compartment is about $1.6 \mathrm{~km}^{2}$. The pond in a compartment bears the name/number of that compartment. Study stations within the study area are shown in Figure 1.

\subsection{Study stations}

Pond 36: $\mathrm{N}^{\circ} 6^{\circ} 23.024$ ', E005 $21.059^{\prime}$. Pond 36 is located along the access earth-road and the forest floor usually submerged during heavy rains.

Pond 52: N06 $21.704^{\prime}$, E005 $19.634^{\prime}$. This station is shaded by tree canopy and ahead of Pond 51. The substratum is sandy and with decaying leaf litter.

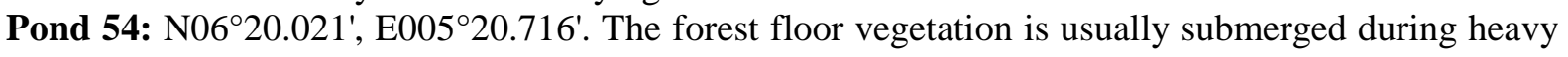
rains. It is shaded by forest tree canopy and the substratum composed of leaf litter and sand.

Pond 61: N06 ${ }^{\circ} 19.998 '$ ', E005 $20.720^{\prime}$. This pond is large and was frequently sampled because of its size, easy accessibility and its retention of water for most part of the year. It is treeless with muddy substratum.

Pond 64: $\mathrm{N}^{\circ} 6^{\circ} 20.480^{\prime}$, E $005^{\circ} 18.115^{\prime}$. The pond is partially shaded and surface is covered by floating macrophyte. The substratum is rich in organic materials.

Pond 90: $\mathrm{N}^{\circ} 6^{\circ} 20.731^{\prime}, \mathrm{E} 005^{\circ} 15.0777^{\prime}$. This station is exposed to direct sunlight. The substratum is clayey.

Pond 94: $\mathrm{N}^{\circ} 6^{\circ} 18.288^{\prime}, \mathrm{E} 005^{\circ} 18.748^{\prime}$. The depth is irregular as a result of elephant-dug holes on the average measure 2.5 meters. This station is shaded by forest tree canopy. 


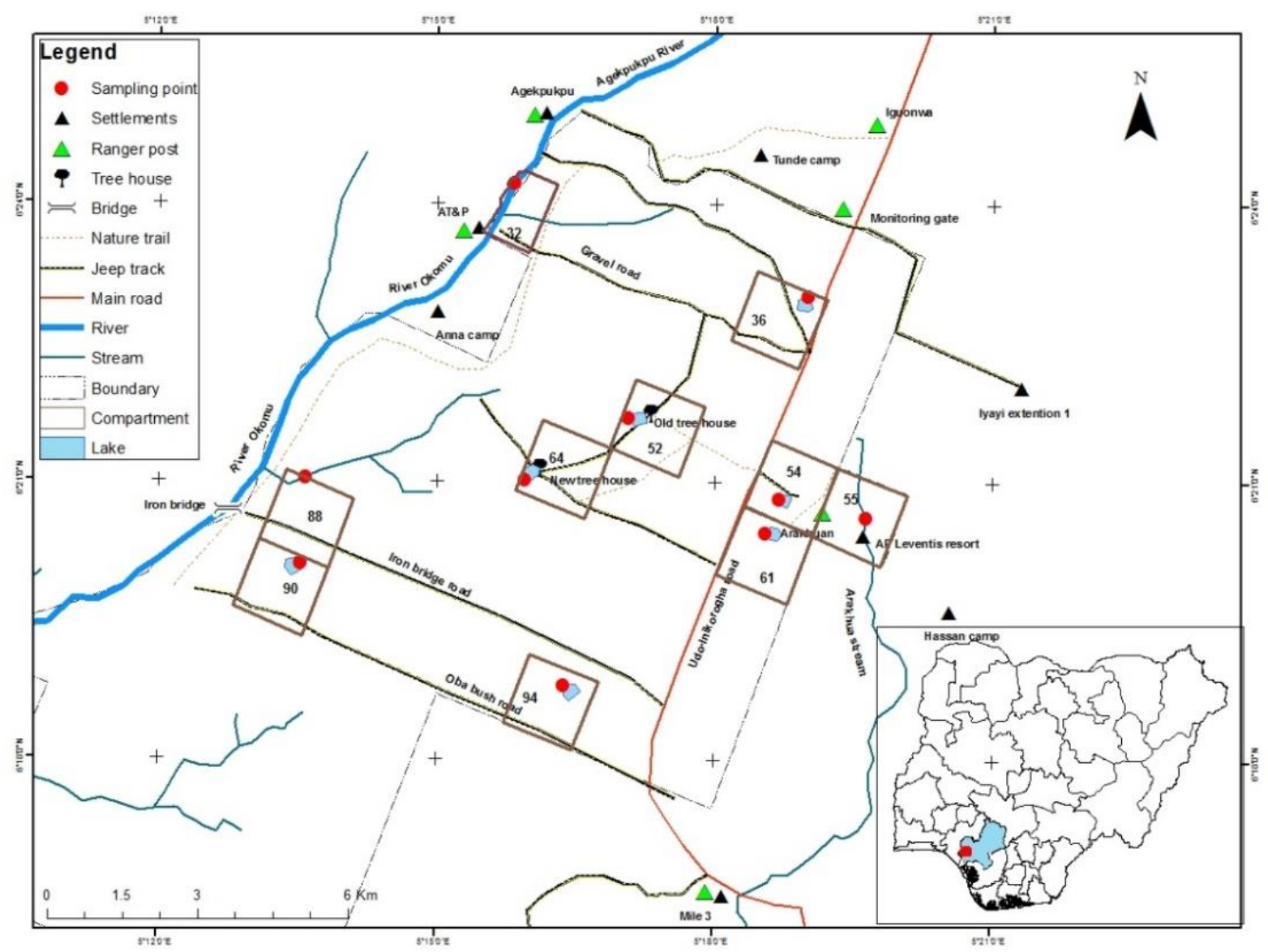

Figure 1: Map showing sampling stations in the study area, Okomu, Benin City, Edo State, Nigeria

Arakhuan Stream: N06 $20.609^{\prime}$, E005 $21.588^{\prime}$. The Stream is clear and shallow with an average depth of $0.6 \mathrm{~m}$ and about $4 \mathrm{~m}$ wide at the point of sampling. The substratum consists of fine sand. It is shaded by dense tree canopy. The Arhakhuan Stream is in compartment 55.

Okomu River (Agekpukpu): N06 24.879', E005 ${ }^{\circ} 17.9966^{\prime}$. The River is about $5 \mathrm{~m}$ wide at the point of sampling. The water is clear with minimal human activities in the area. The Okomu River (Agekpukpu) is in compartment 32.

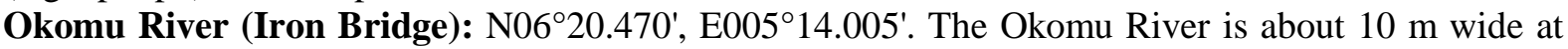
the point of sampling and flows through compartment 88 . The sampled area is situated downstream of the bridge. Generally, surface run-off and organic matter from the surrounding vegetation contributes to the allochthonous input. There are hardly human activities in the area. The Okomu Bridge serves as boundary between the national park and surrounding communities.

\subsection{Analysis of physicochemical parameters}

All containers for sample collection were pre-washed and air-dried. Each sample bottle was rinsed with water from the sampling station before samples were collected into the containers. All samples for physicochemical analysis were preserved and transported to the laboratory in ice-chest. Air and water temperatures were measured in situ using the mercury in glass thermometer (Krisson model-59) (Olomukoro and Dirisu, 2012; Dirisu et al., 2016). To measure water level, a stone to which was attached a calibrated rope was lowered into the water until it reached the bottom and the water level read off for the river system while a meter rule was used for measurement in the stream and ponds because of their shallow nature. Hydrogen ion concentration $(\mathrm{pH})$ Electrical conductivity (EC) and Total dissolved solids (TDS) were measured using the $\mathrm{pH} /$ Conductivity/TDS meter (HACH pH meter sense ion 2 Model). Total suspended solids were determined using the photometric method with HACH UV/VIS Spectrophotometer (model DR/2000). The turbidity value in NTU (Nephelometric Turbidity Units) was determined using the turbidity meter (portable HACH Turbidimeter Model 2100p) $\mathrm{BOD}_{5}$ and DO were determined using Winkler's method (Samples for Dissolved Oxygen were fixed in the field with $1 \mathrm{ml}$ each of Winkler's solution A and B). Chloride was determined by Mohr's method, phosphate and nitrate was determined using HACH spectrophotometer (Model DR 2000). Heavy metals were determined using Atomic Absorption Spectrophotometer. The sampling and 
analysis of physicochemical parameters of the water samples were determined by standard methods as detailed in APHA (1998) and APHA (2005).

\subsection{Statistical analysis}

The non-parametric (Kruskal-Wallis) analysis of variance tests were used (because of unequal sample size) for inter-station comparison at ninety-five and ninety-nine percent $(95 \%$ and $99 \%$ that is, $\mathrm{P}<$ 0.05 and $\mathrm{P}<0.001$ respectively) levels of significance. Significant probability values $(\mathrm{P}-$ values $)$ were obtained for all parameters. The Chi-square goodness of fit test was therefore performed to determine the location of significant differences. Statistical computations of central tendency and dispersion to characterize the physicochemical state of the sampled water bodies were also carried out. Statistical analysis was carried out using SPSS version 20.0. Mean values obtained (Table 1) were thereafter compared with surface water standards in Table 2.

\subsection{Results and Discussion}

The results of physicochemical analysis are presented in Table 1. All physicochemical parameters varied significantly $(\mathrm{P}<0.001)$. The temperature of the Okomu wetland ranged between 23.6 and $38.0^{\circ} \mathrm{C}$ for air temperature and $21.0-38^{\circ} \mathrm{C}$ for water temperature. The highest temperature was recorded in Okomu River (Agekpukpu) and Okomu River (Iron Bridge) and lowest temperature recorded in Arhakhuan Stream. The spatial variations observed in water temperatures were as a result of local climatic conditions, exposure of stations to sunlight and the time of sampling. Surface water is influenced by composition of substrate, vegetation cover, run-off, turbidity, and heat exchange with air (Ogbeibu and Victor 1995). According to (Adebisi, 1981), most tropical freshwaters have a stable temperature regime and seasonal variations ranging between $20-30^{\circ} \mathrm{C}$. The observed temperature in the present study is typical of the tropics and agrees with the findings of Obire et al., (2003). Surface water temperatures were generally lower than ambient air temperatures. Spatial variation significantly impacted water level. In most of the temporary ponds, during some period of the year with little or no rainfall, the water completely dried up and began to retain water at the onset of rains. This agrees with the findings of Ogbeibu and Victor (1995). The highest water level was recorded in the Okomu River (Iron Bridge). Variations in water level are usually associated with the rainfall pattern of the drainage basin (Ikusima et al., 1982). The colour values of the wetland were especially higher in Ponds than in the River and Stream and showed significant spatial variations. The maximum colour value was recorded in pond 61 and the least value in Okomu River (Iron Bridge). Despite the higher values of colour recorded in the ponds, they were within the limit of WHO (World Health Organization) and FMEnv. (Federal Ministry of Environment) the higher colour in ponds of the study area is probably due to allochthonous input from run off, presence of leaf litter and decaying plant and animal remains within the wetland.

Turbidity consists of suspended particles in water and may be caused by organic or inorganic materials. The turbidity values for the Okomu Wetland ranged between $0.2-14.8 \mathrm{mg} / \mathrm{l}$. The World Health Organization (WHO) and Federal Ministry of Environment guidelines for turbidity are 5.00 and 1.0 NTU respectively. Turbidity for this study was higher in the ponds than in the Stream and River. The highest turbidity value was recorded in Pond 61 . Higher turbidity values in the ponds than in the Arhakhuan Stream and Okomu River (Agekpukpu and Iron Bridge) could be as a result of leaf litter and decaying materials including animal remains. Turbidity range of $(0.2-165$ NTU) was recorded for Calabar River (Akpan et al., 2003). The observed turbidity level in this study agrees with the range of 2 - 47 NTU reported by Asonye et al. (2007) for the turbidity of Nigerian Rivers, Streams and Waterways.

The total dissolved solids observed were between $16.7-150.7 \mathrm{mg} / \mathrm{l}$ for the Okomu Wetland and within the range of 12 - 490mg/l reported for Ikpoba River (Ogbeibu and Edutie, 2002, Ogbeibu and Ezeunara, 2002). However, much lower than the range of 1235 - 19846mg/l recorded for Elechi Creek in Port-Harcort Nigeria (Obire et al., 2003). The range documented for Calabar River is 6176mg/l (Akpan et al., 2003). 
Table 1: Spatial variation of physicochemical parameters in the study area (Okomu National Park, Edo State, Nigeria) 2017-2018.

\begin{tabular}{|c|c|c|c|c|c|c|c|c|c|c|c|c|}
\hline 递 & $\begin{array}{l}\text { Pond 36 } \\
(\mathrm{n}=19)\end{array}$ & $\begin{array}{l}\text { Pond 52 } \\
(\mathrm{n}=24)\end{array}$ & $\begin{array}{l}\text { Pond 54 } \\
(\mathrm{n}=14)\end{array}$ & $\begin{array}{c}\text { Pond 61 } \\
(\mathrm{n}=22)\end{array}$ & $\begin{array}{c}\text { Pond 64 } \\
(n=7)\end{array}$ & $\begin{array}{l}\text { Pond 90 } \\
(\mathrm{n}=12)\end{array}$ & $\begin{array}{c}\text { Pond } 94 \\
(n=8)\end{array}$ & $\begin{array}{c}\text { Arhakhuan } \\
\text { Stream } \\
(\mathbf{n}=\mathbf{2 4})\end{array}$ & $\begin{array}{c}\text { Okomu River } \\
\text { (Agekpukpu) } \\
(\mathbf{n}=\mathbf{2 4})\end{array}$ & $\begin{array}{c}\begin{array}{c}\text { Okomu River } \\
\text { (Iron bridge }) \\
(\mathrm{n}=12)\end{array}\end{array}$ & \multirow{2}{*}{ 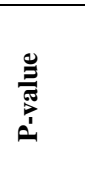 } & \multirow[t]{2}{*}{ 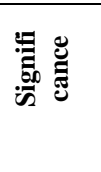 } \\
\hline$\approx$ & \multicolumn{10}{|c|}{ Mean \pm SE $($ Min - Max $)$} & & \\
\hline $\begin{array}{ll}\text { Air } & \text { temp. } \\
\left({ }^{0} \mathrm{C}\right) & \end{array}$ & $\begin{array}{l}26.75 \pm 0.7 \mathrm{~b} \\
(24.0-37.0)\end{array}$ & $\begin{array}{l}27.77 \pm 0.94 \mathrm{a} \\
(25.0-34.0)\end{array}$ & $\begin{array}{l}25.5 \pm 0.74 \mathrm{~b} \\
(25.0-28)\end{array}$ & $\begin{array}{l}26.5 \pm 0.7 \mathrm{~b} \\
(23.0-35.0)\end{array}$ & $\begin{array}{l}25.74 \pm 1.2 \mathrm{~b} \\
(23.6-27.0)\end{array}$ & $\begin{array}{l}26.6 \pm 0.85 b \\
(26.2-37.0)\end{array}$ & $\begin{array}{l}26.0 \pm 2.32 \mathrm{~b} \\
(25.0-33.0)\end{array}$ & $\begin{array}{l}24.6 \pm 1.65 \mathrm{~b} \\
(24.0-33.0)\end{array}$ & $\begin{array}{l}28.2 \pm 1.9 \mathrm{a} \\
(24.5-38.0)\end{array}$ & $\begin{array}{l}28.3 \pm 2.49 a \\
(27.0-36.0)\end{array}$ & 0.000 & $\mathrm{P}<0.001$ \\
\hline $\begin{array}{l}\text { Water Temp. } \\
\left({ }^{0} \mathrm{C}\right)\end{array}$ & $\begin{array}{l}25.2 \pm 0.9 \\
(22-34) \mathrm{b}\end{array}$ & $\begin{array}{l}26.7 \pm 0.69 \\
(23-32.0) \mathrm{a}\end{array}$ & $\begin{array}{l}24.1 \pm 0.5 \\
(23-28) \mathrm{b}\end{array}$ & $\begin{array}{l}25.41 \pm 0.8 \\
(22-33) \mathrm{b}\end{array}$ & $\begin{array}{l}24.14 \pm 0.26 \\
(23-25) \mathrm{b}\end{array}$ & $\begin{array}{l}25.75 \pm 0.9 \\
(25.3-35) \mathrm{b}\end{array}$ & $\begin{array}{l}27 \pm 1.18 \\
(23-32) \mathrm{a}\end{array}$ & $\begin{array}{l}22.92 \pm 0.3 \\
(21-25) \mathrm{B}\end{array}$ & $\begin{array}{l}28.67 \pm 1.0 \\
(23-38) \mathrm{a}\end{array}$ & $\begin{array}{l}27.6 \pm 1.58 \\
(23-38) \mathrm{a}\end{array}$ & 0.000 & $\mathrm{P}<0.001$ \\
\hline $\begin{array}{ll}\begin{array}{l}\text { Water } \\
(\mathrm{m})\end{array} & \text { level } \\
\end{array}$ & $\begin{array}{l}0.39 \pm 0.06 \\
(0.15-0.84) \mathrm{d}\end{array}$ & $\begin{array}{l}0.42 \pm 0.12 \\
(0.36-0.54 d\end{array}$ & $\begin{array}{l}0.83 \pm 0.04 \\
(0.51-0.93) \mathrm{a}\end{array}$ & $\begin{array}{l}0.71 \pm 0.06 \\
(0.3-0.93) \mathrm{b}\end{array}$ & $\begin{array}{l}0.36 \pm 0.1 \\
(0.29-0.9) \mathrm{c}\end{array}$ & $\begin{array}{l}0.72 \pm 0.06 \\
(0.51-0.93) \mathrm{b}\end{array}$ & $\begin{array}{l}0.51 \pm 0.06 \\
(0.3-0.75) \mathrm{d}\end{array}$ & $\begin{array}{c}0.57 \pm 0.05 \\
(0.18-0.9) \mathrm{c}\end{array}$ & $\begin{array}{l}0.27 \pm 0.02 \\
(0.57-0.93) \mathrm{b}\end{array}$ & $\begin{array}{l}0.91 \pm 0.21 \\
(0.78-1.02) \mathrm{a}\end{array}$ & 0.000 & $\mathrm{P}<0.001$ \\
\hline TDS (mg/l) & $\begin{array}{l}41.54 \pm 3.2 \\
(22.2-89.4) \mathrm{b}\end{array}$ & $\begin{array}{l}45.5 \pm 3.1 \\
(21.2-107.1) \mathrm{a}\end{array}$ & $\begin{array}{l}59.81 \pm 6.7 \\
(46.9-143) \mathrm{a}\end{array}$ & $\begin{array}{l}60.7 \pm 7.2 \\
(40.2-150.7) \mathrm{a}\end{array}$ & $\begin{array}{l}43.37 \pm 3.2 \\
(26.4-49.1) \mathrm{a}\end{array}$ & $\begin{array}{l}46.34 \pm 5.9 \\
(25.2-89.2) \mathrm{a}\end{array}$ & $\begin{array}{l}41.39 \pm 3.5 \\
(26.2-49.4) \mathrm{b}\end{array}$ & $\begin{array}{l}37.93 \pm 4.1 \\
(17.6-123.4) \mathrm{b}\end{array}$ & $\begin{array}{l}36.17 \pm 3.5 \\
(16.7-91.1) \mathrm{b}\end{array}$ & $\begin{array}{l}41.3 \pm 5.37 \\
(20.1-74.4) b\end{array}$ & 0.000 & $\mathrm{P}<0.001$ \\
\hline TSS (mg/l) & $\begin{array}{l}7.31 \pm 0.73 \\
(1.1-13.5) \mathrm{b} \\
\end{array}$ & $\begin{array}{l}10.07 \pm 0.2 \\
(7.1-10.6) \mathrm{b}\end{array}$ & $\begin{array}{l}10.64 \pm 0.7 \\
(5.3-12.7) \mathrm{b}\end{array}$ & $\begin{array}{l}15.3 \pm 1.1 \\
(4.4-19.7) \mathrm{a} \\
\end{array}$ & $\begin{array}{l}13.4 \pm 2.13 \\
(7-18.8) \mathrm{c}\end{array}$ & $\begin{array}{l}9.46 \pm 1.12 \\
(6-17.8) \mathrm{b}\end{array}$ & $\begin{array}{l}12.78 \pm 2.0 \\
(7-18.8) \mathrm{b}\end{array}$ & $\begin{array}{l}2.6 \pm 0.403 \\
(0.5-5.7) \mathrm{c}\end{array}$ & $\begin{array}{l}1.63 \pm 0.10 \\
(0.9-2.3) \mathrm{c}\end{array}$ & $\begin{array}{l}2.13 \pm 0.34 \\
(0.9-4) \mathrm{c}\end{array}$ & 0.000 & $\mathrm{P}<0.001$ \\
\hline $\begin{array}{l}\text { Turbidity } \\
\text { (NTU) }\end{array}$ & $\begin{array}{l}5.34 \pm 0.67 \\
(0.5-12.4) b\end{array}$ & $\begin{array}{l}7.25 \pm 0.15 \\
(5.8-8.7) \mathrm{a}\end{array}$ & $\begin{array}{l}7.99 \pm 0.6 \\
(3.2-9.7) \mathrm{a}\end{array}$ & $\begin{array}{l}6.6 \pm 0.8 \\
(3.7-14.8) \mathrm{a}\end{array}$ & $\begin{array}{l}9.46 \pm 1.41 \\
(4.4-13.1) \mathrm{b}\end{array}$ & $\begin{array}{l}6.26 \pm 0.73 \\
(4.3-12.1) \mathrm{b}\end{array}$ & $\begin{array}{l}8.96 \pm 1.43 \\
(4.4-14.1) \mathrm{a}\end{array}$ & $\begin{array}{l}1.21 \pm 0.23 \\
(0.2-3.1) \mathrm{c}\end{array}$ & $\begin{array}{l}0.72 \pm 0.04 \\
(0.4-1.1) \mathrm{c}\end{array}$ & $\begin{array}{l}1.38 \pm 0.29 \\
(0.5-2.8) \mathrm{c}\end{array}$ & 0.000 & $\mathrm{P}<0.001$ \\
\hline $\mathrm{EC}(\mu \mathrm{S} / \mathrm{cm})$ & $\begin{array}{l}85.4 \pm 5.5 \\
(69.4-178.6) \mathrm{b}\end{array}$ & $\begin{array}{l}95.0 \pm 5.7 \\
(40-214.3) \mathrm{a}\end{array}$ & $\begin{array}{l}119.8 \pm 13 \\
(94.4-287.1) \mathrm{a}\end{array}$ & $\begin{array}{l}123.1 \pm 14 \\
(82-307.4) \mathrm{a}\end{array}$ & $\begin{array}{l}92.56 \pm 3.05 \\
(80.8-98.4) \mathrm{c}\end{array}$ & $\begin{array}{l}106.46 \pm 8.6 \\
(75.8-178.5) \mathrm{a}\end{array}$ & $\begin{array}{l}92.4 \pm 2.8 \\
(80.5-99.2) \mathrm{a}\end{array}$ & $\begin{array}{l}78.5 \pm 7.8 \\
(49.9-246.9) \mathrm{b}\end{array}$ & $\begin{array}{l}80.58 \pm 5.92 \\
(54.5-190.3) \mathrm{b}\end{array}$ & $\begin{array}{l}86.9 \pm 9.08 \\
(59.90-148) b\end{array}$ & 0.000 & $\mathrm{P}<0.001$ \\
\hline $\mathrm{pH}$ & $\begin{array}{l}5.4 \pm 0.1 \\
(4.4-5.6) \mathrm{c}\end{array}$ & $\begin{array}{l}5.2 \pm 0.05 \\
(4.6-5.4) \mathrm{d}\end{array}$ & $\begin{array}{l}5.5 \pm 0.13 \\
(5.1-6.4) \mathrm{c}\end{array}$ & $\begin{array}{l}5.6 \pm 0.1 \\
(4.6-6.2) \mathrm{b}\end{array}$ & $\begin{array}{l}6.3 \pm 0.2 \\
(5.2-6.7) \mathrm{a}\end{array}$ & $\begin{array}{l}5.3 \pm 0.13 \\
(4.4-6.4) \mathrm{c}\end{array}$ & $\begin{array}{l}6.2 \pm 0.22 \\
(5.2-6.7) \mathrm{a}\end{array}$ & $\begin{array}{l}5.3 \pm 0.06 \\
(4.5-5.6) \mathrm{c}\end{array}$ & $\begin{array}{l}5.3 \pm 0.08 \\
(4.5-5.7) \mathrm{d}\end{array}$ & $\begin{array}{l}5.2 \pm 0.15 \\
(4.4-5.9) \mathrm{d}\end{array}$ & 0.000 & $\mathrm{P}<0.001$ \\
\hline Salinity (g/l) & $\begin{array}{l}0.037 \pm 0.003 \\
(0.02-0.08) \mathrm{b}\end{array}$ & $\begin{array}{l}0.04 \pm 0.003 \\
(0.02-0.097) \mathrm{a}\end{array}$ & $\begin{array}{l}0.05 \pm 0.01 \\
(0.04-0.13) \mathrm{a}\end{array}$ & $\begin{array}{l}0.06 \pm 0.01 \\
(0.04-0.14) \mathrm{a}\end{array}$ & $\begin{array}{l}0.04 \pm 0.003 \\
(0.02-0.04) \mathrm{a}\end{array}$ & $\begin{array}{l}0.04 \pm 0.005 \\
(0.02-0.08) \mathrm{a}\end{array}$ & $\begin{array}{l}0.04 \pm 0.003 \\
(0.02-0.04) \mathrm{b}\end{array}$ & $\begin{array}{l}0.03 \pm 0.004 \\
(0.02-0.112) \mathrm{b}\end{array}$ & $\begin{array}{l}0.033 \pm 0.03 \\
(0.02-0.086) \mathrm{b}\end{array}$ & $\begin{array}{l}0.04 \pm 0.01 \\
(0.02-0.07) \mathrm{b}\end{array}$ & 0.000 & $\mathrm{P}<0.001$ \\
\hline DO (mg/l) & $\begin{array}{l}1.91 \pm 0.23 \\
(1.1-3.7) \mathrm{b}\end{array}$ & $\begin{array}{l}1.93 \pm 0.17 \\
(1.1-4.2) \mathrm{b}\end{array}$ & $\begin{array}{l}2.8 \pm 0.23 \\
(1.5-3.7) \mathrm{a}\end{array}$ & $\begin{array}{l}3.0 \pm 0.14 \\
(1.9-3.8) \mathrm{a}\end{array}$ & $\begin{array}{l}2.5 \pm 0.11 \\
(2-2.8) \mathrm{c}\end{array}$ & $\begin{array}{l}2.44 \pm 0.19 \\
(1.5-3.2) \mathrm{a}\end{array}$ & $\begin{array}{l}2.45 \pm 0.12 \\
(2-2.9) \mathrm{a}\end{array}$ & $\begin{array}{l}1.54 \pm 0.26 \\
(0.7-5.5) \mathrm{b}\end{array}$ & $\begin{array}{l}2.13 \pm 0.23 \\
(1-5.4) \mathrm{b}\end{array}$ & $\begin{array}{l}2.68 \pm 0.37 \\
(1.5-5) \mathrm{a}\end{array}$ & 0.000 & $\mathrm{P}<0.001$ \\
\hline $\mathrm{BOD}_{5}(\mathrm{mg} / \mathrm{l})$ & $\begin{array}{l}0.94 \pm 0.10 \\
(0.6-2) \mathrm{b}\end{array}$ & $\begin{array}{l}1.07 \pm 0.08 \\
(0.7-2.1) \mathrm{b}\end{array}$ & $\begin{array}{l}1.12 \pm 0.09 \\
(0.8-1.9) \mathrm{b}\end{array}$ & $\begin{array}{l}1.91 \pm 0.2 \\
(0.8-3.8) \mathrm{a}\end{array}$ & $\begin{array}{l}1.34 \pm 0.13 \\
(0.8-1.6) \mathrm{c}\end{array}$ & $\begin{array}{l}1.2 \pm 0.13 \\
(0.8-1.8) \mathrm{b}\end{array}$ & $\begin{array}{l}1.26 \pm 0.13 \\
(0.8-1.6) \mathrm{b}\end{array}$ & $\begin{array}{l}1.21 \pm 0.16 \\
(0.5-2.3) \mathrm{b}\end{array}$ & $\begin{array}{l}0.99 \pm 0.09 \\
(0.5-1.5) \mathrm{b}\end{array}$ & $\begin{array}{l}1.0 \pm 0.15 \\
(0.5-1.9) \mathrm{b}\end{array}$ & 0.000 & $\mathrm{P}<0.001$ \\
\hline COD (mg/l) & $\begin{array}{c}33.07 \pm 6.66 \\
(8-109.4)\end{array}$ & $\begin{array}{l}32.2 \pm 6.1 \\
(10.4-120.2) \mathrm{b} \\
\end{array}$ & $\begin{array}{l}32.85 \pm 6.33 \\
(13.3-86.4) \mathrm{b}\end{array}$ & $\begin{array}{l}33.7 \pm 5.1 \\
(15.8-88.2) \mathrm{b}\end{array}$ & $\begin{array}{l}23.46 \pm 2.93 \\
(17.3-33.6) \mathrm{a} \\
\end{array}$ & $\begin{array}{l}41.14 \pm 6.19 \\
(4.3-78.5) \mathrm{a} \\
\end{array}$ & $\begin{array}{l}24.71 \pm 2.83 \\
(17.3-33.6) \mathrm{b} \\
\end{array}$ & $\begin{array}{l}11.61 \pm 1.081 \\
(7.2-30.2) \mathrm{c}\end{array}$ & $\begin{array}{l}10.30 \pm 1.46 \\
(1.6-22.3) \mathrm{c}\end{array}$ & $\begin{array}{l}28.09 \pm 10.7 \\
(1.5-90.7) \mathrm{b}\end{array}$ & 0.000 & $\mathrm{P}<0.001$ \\
\hline $\mathrm{HCO}_{3}(\mathrm{mg} / \mathrm{l})$ & $\begin{array}{l}123.7 \pm 19.03 \\
(30.5-293.3) \mathrm{c}\end{array}$ & $\begin{array}{l}166.18 \pm 20.64 \\
(30.5-363.2) b\end{array}$ & $\begin{array}{l}235.8 \pm 55.6 \\
(36.4-490) \mathrm{a}\end{array}$ & $\begin{array}{l}227.9 \pm 34.62 \\
(46.2-593.6) \mathrm{a}\end{array}$ & $\begin{array}{l}190.3 \pm 15.6 \\
(135.3- \\
250.1) \mathrm{c} \\
\end{array}$ & $\begin{array}{l}240.28 \pm 28.45 \\
(89.9-399.5) \mathrm{a}\end{array}$ & $\begin{array}{l}197.4 \pm 15.3 \\
(135.3- \\
250.1) \mathrm{b} \\
\end{array}$ & $\begin{array}{l}75.0 \pm 10.23 \\
(23.8-264.7) \mathrm{c}\end{array}$ & $\begin{array}{r}118.7 \pm 11.57 \\
(61-189.3) \mathrm{c}\end{array}$ & $\begin{array}{l}99.08 \pm 11.75 \\
(60.9-189.1) \mathrm{c}\end{array}$ & 0.000 & $\mathrm{P}<0.001$ \\
\hline $\mathrm{Cl}(\mathrm{mg} / \mathrm{l})$ & $\begin{array}{l}108.4 \pm 10.56 \\
(70.1-206.1) \mathrm{c}\end{array}$ & $\begin{array}{l}114.6 \pm 10.79 \\
(70.9-227.8) b\end{array}$ & $\begin{array}{l}127.1 \pm 16.3 \\
(69.9-234.6) \mathrm{a}\end{array}$ & $\begin{array}{l}133.4 \pm 12.01 \\
(69.9-245.3) \mathrm{a}\end{array}$ & $\begin{array}{l}125.03 \pm 10.03 \\
(106.4- \\
162.7) \mathrm{b} \\
\end{array}$ & $\begin{array}{l}148.08 \pm 12.7 \\
(106.4-236.6) \mathrm{a}\end{array}$ & $\begin{array}{l}124.7 \pm 8.66 \\
(105.4- \\
162.7) \mathrm{a} \\
\end{array}$ & $\begin{array}{l}85.5 \pm 7.4 \\
(52.7-197.2) \mathrm{c}\end{array}$ & $\begin{array}{l}109.0 \pm 4.8 \\
(85.7-175.2) \mathrm{c}\end{array}$ & $\begin{array}{l}120.13 \pm 8.44 \\
(88.6-180.3) b\end{array}$ & 0.001 & $\mathrm{P}<0.001$ \\
\hline $\mathrm{NO}_{2}(\mathrm{mg} / \mathrm{l})$ & $\begin{array}{l}0.18 \pm 0.01 \\
(0.13-0.25) \mathrm{b} \\
\end{array}$ & $\begin{array}{l}0.21 \pm 0.013 \\
(0.13-0.28) \mathrm{b} \\
\end{array}$ & $\begin{array}{l}0.23 \pm 0.02 \\
(0.16-0.36) \mathrm{a} \\
\end{array}$ & $\begin{array}{l}0.32 \pm 0.04 \\
(0.19-0.59) \mathrm{a} \\
\end{array}$ & $\begin{array}{l}0.33 \pm 0.095 \\
(0.03-0.54) \mathrm{b} \\
\end{array}$ & $\begin{array}{l}0.199 \pm 0.03 \\
(0.13-0.53) \mathrm{b} \\
\end{array}$ & $\begin{array}{l}0.307 \pm 0.09 \\
(0.03-0.54) \mathrm{a} \\
\end{array}$ & $\begin{array}{l}0.126 \pm 0.02 \\
(0.02-0.34) \mathrm{b} \\
\end{array}$ & $\begin{array}{l}0.14 \pm 0.03 \\
(0.02-0.33) \mathrm{b} \\
\end{array}$ & $\begin{array}{l}0.09 \pm 0.024 \\
(0.04-0.33) \mathrm{a} \\
\end{array}$ & 0.000 & $\mathrm{P}<0.001$ \\
\hline $\mathrm{NO}_{3}(\mathrm{mg} / \mathrm{l})$ & $\begin{array}{l}0.78 \pm 0.06 \\
(0.51-1.10) \mathrm{b}\end{array}$ & $\begin{array}{l}0.95 \pm 0.08 \\
(0.54-2.2) \mathrm{a}\end{array}$ & $\begin{array}{l}0.96 \pm 0.12 \\
(0.68-2.19) \mathrm{a} \\
\end{array}$ & $\begin{array}{l}1.39 \pm 0.14 \\
(0.78-2.34) \mathrm{a} \\
\end{array}$ & $\begin{array}{l}1.36 \pm 0.36 \\
(0.23-2.13) \mathrm{c}\end{array}$ & $\begin{array}{l}0.95 \pm 0.15 \\
(0.54-2.12) \mathrm{a} \\
\end{array}$ & $\begin{array}{l}1.27 \pm 0.34 \\
(0.23-2.22) \mathrm{a} \\
\end{array}$ & $\begin{array}{l}0.55 \pm 0.09 \\
(0.12-1.69) \mathrm{b}\end{array}$ & $\begin{array}{l}0.58 \pm 0.12 \\
(0.11-1.38) \mathrm{b}\end{array}$ & $\begin{array}{l}0.48 \pm 0.12 \\
(0.15-1.32) \mathrm{c}\end{array}$ & 0.000 & $\mathrm{P}<0.001$ \\
\hline $\mathrm{NH}_{4} \mathrm{~N}(\mathrm{mg} / \mathrm{l})$ & $\begin{array}{l}0.22 \pm 0.02 \\
(0.13-0.41) \mathrm{b}\end{array}$ & $\begin{array}{l}0.35 \pm 0.02 \\
(0.26-0.61) \mathrm{b}\end{array}$ & $\begin{array}{l}0.51 \pm 0.06 \\
(0.33-1.12) \mathrm{b}\end{array}$ & $\begin{array}{l}0.89 \pm 0.15 \\
(0.35-2.11) \mathrm{a} \\
\end{array}$ & $\begin{array}{l}1.1 \pm 0.3 \\
(0.25-1.7) \mathrm{b}\end{array}$ & $\begin{array}{l}0.43 \pm 0.12 \\
(0.19-1.74) \mathrm{b}\end{array}$ & $\begin{array}{l}0.99 \pm 0.28 \\
(0.25-1.74) \mathrm{a} \\
\end{array}$ & $\begin{array}{l}0.15 \pm 0.03 \\
(0.06-0.73) \mathrm{c}\end{array}$ & $\begin{array}{l}0.21 \pm 0.03 \\
(0.04-0.40) \mathrm{b}\end{array}$ & $\begin{array}{l}0.19 \pm 0.02 \\
(0.12-0.35) \mathrm{b}\end{array}$ & 0.000 & $\mathrm{P}<0.001$ \\
\hline $\mathrm{SO}_{4}(\mathrm{mg} / \mathrm{l})$ & $\begin{array}{l}0.28 \pm 0.03 \\
(0.18-0.53)\end{array}$ & $\begin{array}{l}0.39 \pm 0.03 \\
(0.25-0.72)\end{array}$ & $\begin{array}{l}0.55 \pm 0.09 \\
(0.28-1.34)\end{array}$ & $\begin{array}{l}1.36 \pm 1.25 \\
(0.4-2.96)\end{array}$ & $\begin{array}{l}1.12 \pm 0.27 \\
(0.37-1.69)\end{array}$ & $\begin{array}{l}0.53 \pm 0.11 \\
(0.30-1.69)\end{array}$ & $\begin{array}{l}1.03 \pm 0.25 \\
(0.36-1.7)\end{array}$ & $\begin{array}{l}0.18 \pm 0.03 \\
(0.05-0.80)\end{array}$ & $\begin{array}{l}0.39 \pm 0.04 \\
(0.17-0.67)\end{array}$ & $\begin{array}{l}0.32 \pm 0.04 \\
(0.21-0.67)\end{array}$ & 0.000 & $\mathrm{P}<0.001$ \\
\hline $\mathrm{P}(\mathrm{mg} / \mathrm{l})$ & $\begin{array}{l}0.42 \pm 0.06 \\
(0.14-0.84)\end{array}$ & $\begin{array}{l}0.78 \pm 0.10 \\
(0.22-1.81)\end{array}$ & $\begin{array}{l}1.004 \pm 0.12 \\
(0.56-2.09)\end{array}$ & $\begin{array}{l}4.3 \pm 4.2 \\
(0.71-9.2)\end{array}$ & $\begin{array}{c}1.13 \pm 0.25 \\
(0.4-1.67)\end{array}$ & $\begin{array}{l}0.88 \pm 0.17 \\
(0.43-1.85)\end{array}$ & $\begin{array}{l}1.04 \pm 0.24 \\
(0.39-1.67)\end{array}$ & $\begin{array}{l}0.29 \pm 0.06 \\
(0.09-1.27)\end{array}$ & $\begin{array}{l}0.27 \pm 0.05 \\
(0.09-1.23)\end{array}$ & $\begin{array}{l}0.35 \pm 0.04 \\
(0.20-0.56)\end{array}$ & 0.000 & $\mathrm{P}<0.001$ \\
\hline
\end{tabular}




\begin{tabular}{|c|c|c|c|c|c|c|c|c|c|c|c|c|}
\hline है & $\begin{array}{l}\text { Pond 36 } \\
(n=19)\end{array}$ & $\begin{array}{l}\text { Pond 52 } \\
(\mathrm{n}=\mathbf{2 4})\end{array}$ & $\begin{array}{l}\text { Pond 54 } \\
(n=14)\end{array}$ & $\begin{array}{c}\begin{array}{c}\text { Pond 61 } \\
(n=22)\end{array} \\
\text { (n) }\end{array}$ & $\begin{array}{c}\text { Pond 64 } \\
(n=7)\end{array}$ & $\begin{array}{l}\text { Pond 90 } \\
(n=12)\end{array}$ & $\begin{array}{c}\text { Pond 94 } \\
(\mathrm{n}=8)\end{array}$ & $\begin{array}{l}\text { Arhakhuan } \\
\text { Stream } \\
(\mathbf{n}=\mathbf{2 4})\end{array}$ & $\begin{array}{c}\text { Okomu River } \\
\text { (Agekpukpu) } \\
(\mathrm{n}=\mathbf{2 4})\end{array}$ & $\begin{array}{c}\text { Okomu River } \\
\text { (Iron bridge) } \\
\quad(n=12)\end{array}$ & A & 巻 \\
\hline $\mathrm{Na}(\mathrm{mg} / \mathrm{l})$ & $\begin{array}{l}0.64 \pm 0.04 \\
(0.42-1.13) \mathrm{b}\end{array}$ & $\begin{array}{l}0.76 \pm 0.05 \\
(0.53-1.46) \mathrm{b} \\
\end{array}$ & $\begin{array}{l}1.25 \pm 0.19 \\
(0.56-2.71) \mathrm{a} \\
\end{array}$ & $\begin{array}{l}1.18 \pm 0.122 \\
(0.71-2.72) \mathrm{a} \\
\end{array}$ & $\begin{array}{l}0.82 \pm 0.09 \\
(0.47-1.01) \mathrm{c}\end{array}$ & $\begin{array}{l}0.90 \pm 0.17 \\
(0.47-2.02) \mathrm{a}\end{array}$ & $\begin{array}{l}0.78 \pm 0.09 \\
(0.46-1.02) \mathrm{b}\end{array}$ & $\begin{array}{l}0.45 \pm 0.056 \\
(0.24-1.65) \mathrm{c}\end{array}$ & $\begin{array}{l}0.57 \pm 0.07 \\
(0.2-1.56) \mathrm{b}\end{array}$ & $\begin{array}{l}0.63 \pm 0.09 \\
(0.31-1.11) \mathrm{b} \\
\end{array}$ & 0.000 & $\mathrm{P}<0.001$ \\
\hline $\bar{K}(\mathrm{mg} / \mathbf{l})$ & $\begin{array}{l}0.12 \pm 0.02 \\
(0.06-0.27) \mathrm{b} \\
\end{array}$ & $\begin{array}{l}0.17 \pm 0.02 \\
(0.08-0.4) \mathrm{b} \\
\end{array}$ & $\begin{array}{l}0.43 \pm 0.09 \\
(0.14-1.32) \mathrm{a} \\
\end{array}$ & $\begin{array}{l}0.35 \pm 0.05 \\
(0.16-0.95) \mathrm{a} \\
\end{array}$ & $\begin{array}{l}0.17 \pm 0.03 \\
(0.06-0.24) \mathrm{b} \\
\end{array}$ & $\begin{array}{l}0.18 \pm 0.05 \\
(0.05-0.56) b \\
\end{array}$ & $\begin{array}{l}0.16 \pm 0.03 \\
(0.06-0.22) \mathrm{b} \\
\end{array}$ & $\begin{array}{l}0.08 \pm 0.02 \\
(0.05-0.52) b\end{array}$ & $\begin{array}{l}0.12 \pm 0.01 \\
(0.03-0.33) \mathrm{b} \\
\end{array}$ & $\begin{array}{l}0.1 \pm 0.01 \\
(0.05-0.19) \mathrm{b} \\
\end{array}$ & 0.000 & $\mathrm{P}<0.001$ \\
\hline Ca (mg/l) & $\begin{array}{l}1.64 \pm 0.09 \\
(1.24-2.57) \mathrm{c}\end{array}$ & $\begin{array}{l}2.07 \pm 0.11 \\
(1.57-3.19) \mathrm{b} \\
\end{array}$ & $\begin{array}{l}3.03 \pm 0.32 \\
(1.91-4.88) \mathrm{a} \\
\end{array}$ & $\begin{array}{l}3.22 \pm 0.23 \\
(2.05-5.88) \mathrm{a} \\
\end{array}$ & $\begin{array}{l}2.67 \pm 0.27 \\
(1.35-3.19) \mathrm{c} \\
\end{array}$ & $\begin{array}{l}2.29 \pm 0.38 \\
(1.34-4.9) \mathrm{b} \\
\end{array}$ & $\begin{array}{l}2.51 \pm 0.28 \\
(1.35-3.19) \mathrm{b} \\
\end{array}$ & $\begin{array}{l}1.31 \pm 0.07 \\
(0.88-2.43) \mathrm{c} \\
\end{array}$ & $\begin{array}{l}1.89 \pm 0.15 \\
(1.05-2.85) \mathrm{c} \\
\end{array}$ & $\begin{array}{l}1.72 \pm 0.17 \\
(1.15-2.83) \mathrm{c}\end{array}$ & 0.000 & $\mathrm{P}<0.001$ \\
\hline $\mathrm{Mg}(\mathrm{mg} / \mathrm{l})$ & $\begin{array}{l}0.70 \pm 0.06 \\
(0.28-1.17) \mathrm{c}\end{array}$ & $\begin{array}{l}0.901 \pm 0.05 \\
(0.52-1.84) \mathrm{b} \\
\end{array}$ & $\begin{array}{l}1.3 \pm 0.17 \\
(0.84-3.1) \mathrm{a} \\
\end{array}$ & $\begin{array}{l}1.3 \pm 0.11 \\
(0.75-2.54) \mathrm{a} \\
\end{array}$ & $\begin{array}{l}0.95 \pm 0.18 \\
(0.38-1.33) \mathrm{c} \\
\end{array}$ & $\begin{array}{l}0.97 \pm 0.14 \\
(0.54-1.87) \mathrm{a} \\
\end{array}$ & $\begin{array}{l}0.90 \pm 0.16 \\
(0.38-1.33) \mathrm{b} \\
\end{array}$ & $\begin{array}{l}0.57 \pm 0.09 \\
(0.17-2.37) \mathrm{c} \\
\end{array}$ & $\begin{array}{l}0.50 \pm 0.086 \\
(0.13-1.46) \mathrm{c} \\
\end{array}$ & $\begin{array}{l}0.47 \pm 0.1 \\
(0.13-1.01) \mathrm{c} \\
\end{array}$ & 0.000 & $\mathrm{P}<0.001$ \\
\hline \multicolumn{13}{|c|}{$\left(\mathbf{x 1 0 ^ { - 1 }}\right) \mathrm{mg} / \mathrm{l}$} \\
\hline $\mathrm{Fe}$ & $\begin{array}{l}8.7 \pm 0.4 \mathrm{c} \\
(1.9-9.1)\end{array}$ & $\begin{array}{l}11.3 \pm 0.1 \\
(10.7-12)\end{array}$ & $\begin{array}{l}17.5 \pm 0.6 \mathrm{a} \\
(11.9-18.9) \\
\end{array}$ & $\begin{array}{l}10.9 \pm 0.0 \mathrm{~b} \\
(11-11.2)\end{array}$ & $\begin{array}{l}11 \pm 0.03 \mathrm{~b} \\
(10.8-11.0)\end{array}$ & $\begin{array}{l}8.1 \pm 0.01 \mathrm{c} \\
(7.6-8.9)\end{array}$ & $\begin{array}{l}8.1 \pm 0.014 \mathrm{c} \\
(7.60-8.90) \\
\end{array}$ & $\begin{array}{l}6.8 \pm 0.01 \mathrm{~d} \\
(6.4-7.2)\end{array}$ & $\begin{array}{l}6.7 \pm 0.04 d \\
(6.2-6.8)\end{array}$ & $\begin{array}{l}6.5 \pm 0.07 \mathrm{~d} \\
(5.8-6.8)\end{array}$ & 0.000 & $\mathrm{P}<0.001$ \\
\hline $\mathrm{Mn}$ & $\begin{array}{l}1.02 \pm 0.0 \mathrm{c} \\
(0.10-1.1) \\
\end{array}$ & $\begin{array}{l}0.16 \pm 0.002 \mathrm{a} \\
(0.14-0.178)\end{array}$ & $\begin{array}{l}1.00 \pm 0.013 \mathrm{c} \\
(0.071-0.2) \\
\end{array}$ & $\begin{array}{l}0.13 \pm 0.003 b \\
(0.11-0.144)\end{array}$ & $\begin{array}{l}0.13 \pm 0.005 b \\
(0.11-0.143) \\
\end{array}$ & $\begin{array}{l}0.09 \pm 0.001 \mathrm{c} \\
(0.08-0.094) \\
\end{array}$ & $\begin{array}{l}0.09 \pm 0.001 \mathrm{c} \\
(0.086-0.09) \\
\end{array}$ & $\begin{array}{l}0.08 \pm 0.001 \mathrm{~d} \\
(0.06-0.083) \\
\end{array}$ & $\begin{array}{l}0.06 \pm 0.00 \mathrm{e} \\
(0.054-0.06)\end{array}$ & $\begin{array}{l}0.04 \pm 0.001 \mathrm{f} \\
(0.04-0.045)\end{array}$ & 0.000 & $\mathrm{P}<0.001$ \\
\hline $\mathrm{Cu}$ & $\begin{array}{l}0.51 \pm 0.0 \mathrm{~A} \\
(0.1-0.05)\end{array}$ & $\begin{array}{l}0.07 \pm 0.003 b \\
(0.031-0.09)\end{array}$ & $\begin{array}{l}0.1 \pm 0.004 \mathrm{C} \\
(0.031-0.08)\end{array}$ & $\begin{array}{l}0.07 \pm 0.004 \mathrm{~b} \\
(0.03-0.085)\end{array}$ & $\begin{array}{l}0.07 \pm 0.009 b \\
(0.03-0.088)\end{array}$ & $\begin{array}{l}0.040 \pm 0.00 \mathrm{~b} \\
(0.036-0.04)\end{array}$ & $\begin{array}{l}0.04 \pm 0.001 \mathrm{~b} \\
(0.036-0.04)\end{array}$ & $\begin{array}{l}0.034 \pm 0.00 \mathrm{~b} \\
(0.021-0.04)\end{array}$ & $\begin{array}{l}0.02 \pm 0.00 \mathrm{C} \\
(0.02-0.025)\end{array}$ & $\begin{array}{l}0.02 \pm 0.00 \mathrm{C} \\
(0.018-0.018)\end{array}$ & 0.000 & $\mathrm{P}<0.001$ \\
\hline $\mathrm{Zn}$ & $\begin{array}{l}2.41 \pm 0.0 \\
(2.4-0.24) \mathrm{c} \\
\end{array}$ & $\begin{array}{l}3.9 \pm 0.24 \\
(1.0-6.0) \mathrm{b} \\
\end{array}$ & $\begin{array}{l}4.42 \pm 0.00 \mathrm{a} \\
(4.39-4.46) \\
\end{array}$ & $\begin{array}{l}2.70 \pm 0.13 \\
(1.00-4.00) \mathrm{c}\end{array}$ & $\begin{array}{l}2.81 \pm 0.09 \\
(2.30-3.0) \mathrm{c}\end{array}$ & $\begin{array}{l}1.93 \pm 0.0 \\
(1.92-1.9) \mathrm{d} \\
\end{array}$ & $\begin{array}{l}1.93 \pm 0.00 \\
(1.92-1.94) \mathrm{d}\end{array}$ & $\begin{array}{l}1.25 \pm 0.00 \\
(1.2-1.3) \mathrm{e}\end{array}$ & $\begin{array}{l}1.11 \pm 0.01 \\
(1.00-1.30) \mathrm{e} \\
\end{array}$ & $\begin{array}{l}0.93 \pm 0.00 \\
(0.93-0.93) \mathrm{e}\end{array}$ & 0.000 & $\mathrm{P}<0.001$ \\
\hline $\mathrm{Pb}$ & $\begin{array}{l}0.17 \pm 0.0 \\
(0.15-0.2) \mathrm{b} \\
\end{array}$ & $\begin{array}{l}0.15 \pm 0.01 \\
(0.1-2.9) \mathrm{b} \\
\end{array}$ & $\begin{array}{l}0.24 \pm 0.00 \\
(0.23-0.28) \mathrm{a} \\
\end{array}$ & $\begin{array}{l}0.14 \pm 0.01 \\
(0.10-0.26) \mathrm{b} \\
\end{array}$ & $\begin{array}{l}0.13 \pm 0.02 \\
(0.1-0.23) \mathrm{b} \\
\end{array}$ & $\begin{array}{c}0.11 \pm 0.00 \\
(0.1-0.11) \mathrm{b} \\
\end{array}$ & $\begin{array}{l}0.11 \pm 0.0 \\
(0.11-0.11) \mathrm{b} \\
\end{array}$ & $\begin{array}{l}0.10 \pm 0.00 \\
(0.01-0.1) \mathrm{c} \\
\end{array}$ & $\begin{array}{l}0.08 \pm 0.00 \\
(0.05-0.08) \mathrm{c}\end{array}$ & $\begin{array}{l}0.05 \pm 0.00 \\
(0.05-0.05) \mathrm{c} \\
\end{array}$ & 0.000 & $\mathrm{P}<0.001$ \\
\hline $\mathrm{Cd}$ & $\begin{array}{l}0.08 \pm 0.0 \\
(0.03-0.1) \mathrm{b}\end{array}$ & $\begin{array}{l}0.08 \pm 0.01 \\
(0.05-0.17) \mathrm{b}\end{array}$ & $\begin{array}{l}0.14 \pm 0.00 \\
(0.12-0.18) \mathrm{a}\end{array}$ & $\begin{array}{l}0.08 \pm 0.01 \\
(0.05-0.17) \mathrm{b}\end{array}$ & $\begin{array}{l}0.10 \pm 0.02 \\
(0.05-0.17) \mathrm{b}\end{array}$ & $\begin{array}{l}0.05 \pm 0.00 \\
(0.05-0.05) \mathrm{c}\end{array}$ & $\begin{array}{l}0.05 \pm 0.00 \\
(0.05-0.05) \mathrm{c}\end{array}$ & $\begin{array}{l}0.05 \pm 0.01 \\
(0.01-0.1) \mathrm{c}\end{array}$ & $\begin{array}{l}0.03 \pm 0.00 \\
(0.01-0.05) \mathrm{c}\end{array}$ & $\begin{array}{l}0.01 \pm 0.00 \\
(0.01-0.01) \mathrm{c}\end{array}$ & 0.000 & $\mathrm{P}<0.001$ \\
\hline $\mathrm{Cr}$ & $\begin{array}{l}0.2 \pm 0.01 \\
(0.1-0.22) \mathrm{b}\end{array}$ & $\begin{array}{l}0.19 \pm 0.02 \\
(0.10-0.34) \mathrm{b} \\
\end{array}$ & $\begin{array}{l}0.59 \pm 0.26 \\
(0.20-4.0) \mathrm{a} \\
\end{array}$ & $\begin{array}{l}0.19 \pm 0.02 \\
(0.1-0.32) b\end{array}$ & $\begin{array}{l}0.18 \pm 0.03 \\
(0.10-0.32) \mathrm{b} \\
\end{array}$ & $\begin{array}{l}0.10 \pm 0.00 \\
(0.10-0.1) \mathrm{b} \\
\end{array}$ & $\begin{array}{l}0.10 \pm 0.00 \\
(0.10-0.10) \mathrm{b} \\
\end{array}$ & $\begin{array}{l}0.07 \pm 0.00 \\
(0.06-0.1) \mathrm{b} \\
\end{array}$ & $\begin{array}{l}0.10 \pm 0.00 \\
(0.10-0.10) \mathrm{b}\end{array}$ & $\begin{array}{l}0.09 \pm 0.00 \\
(0.09-0.09) \mathrm{b} \\
\end{array}$ & 0.000 & $\mathrm{P}<0.001$ \\
\hline $\mathrm{Ni}$ & $\begin{array}{l}0.07 \pm 0.0 \\
(0.05-0.1) \mathrm{b}\end{array}$ & $\begin{array}{l}0.06 \pm 0.01 \\
(0.02-0.15) b\end{array}$ & $\begin{array}{l}0.12 \pm 0.01 \\
(0.02-0.14) \mathrm{a}\end{array}$ & $\begin{array}{l}0.05 \pm 0.01 \\
(0.02-0.15) \mathrm{b}\end{array}$ & $\begin{array}{l}0.07 \pm 0.02 \\
(0.02-0.15) \mathrm{b}\end{array}$ & $\begin{array}{l}0.04 \pm 0.0 \\
(0.02-0.04) \mathrm{c}\end{array}$ & $\begin{array}{l}0.04 \pm 0.00 \\
(0.04-0.04) \mathrm{c}\end{array}$ & $\begin{array}{l}0.03 \pm 0.00 \\
(0.02-0.03) \mathrm{c}\end{array}$ & $\begin{array}{l}0.03 \pm 0.00 \\
(0.01-0.06) \mathrm{c}\end{array}$ & $\begin{array}{l}0.02 \pm 0.00 \\
(0.02-0.02) \mathrm{c}\end{array}$ & 0.000 & $\mathrm{P}<0.001$ \\
\hline $\mathrm{V}$ & $\begin{array}{l}0.05 \pm 0.0 \\
(0.03-0.07) \mathrm{b}\end{array}$ & $\begin{array}{l}0.10 \pm 0.0 \\
(0.08-0.11) \mathrm{a}\end{array}$ & $\begin{array}{l}0.10 \pm 0.0 \\
(0.1-0.11) \mathrm{a} \\
\end{array}$ & $\begin{array}{l}0.1 \pm 0.0 \\
(0.04-0.11) \mathrm{a}\end{array}$ & $\begin{array}{l}0.10 \pm 0.00 \\
(0.10-0.11) \mathrm{a} \\
\end{array}$ & $\begin{array}{l}0.03 \pm 0.00 \\
(0.03-0.03) \mathrm{c}\end{array}$ & $\begin{array}{l}0.03 \pm 0.00 \\
(0.01-0.04) \mathrm{c}\end{array}$ & $\begin{array}{l}0.03 \pm 0.00 \\
(0.02-0.03) \mathrm{d}\end{array}$ & $\begin{array}{l}0.02 \pm 0.00 \\
(0.01-0.04) \mathrm{d}\end{array}$ & $\begin{array}{l}0.02 \pm 0.00 \\
(0.02-0.02) \mathrm{d}\end{array}$ & 0.000 & $\mathrm{P}<0.001$ \\
\hline
\end{tabular}

NOTE: $\mathrm{P}<0.001=$ Very highly significantly different

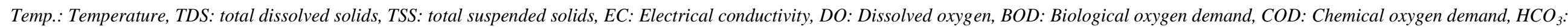

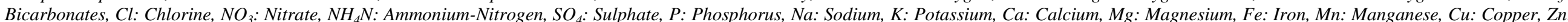
Zinc, Pb: Lead, Cd: Cadmium, Cr: Chromium, Ni: Nickel, V: Vanadium. 
Table 2: Some parameters with FMEnv. 1999 and WHO 1999 Standards

\begin{tabular}{|c|c|c|}
\hline Parameters & FMEnv. 1999 & WHO, 1999 \\
\hline \multicolumn{3}{|c|}{ Water temperature $\left({ }^{0} \mathrm{C}\right)$} \\
\hline TDS (mg/l) & 500 & 500 \\
\hline TSS (mg/l) & $<10$ & \\
\hline Turbidity (NTU) & 1.0 & 5 \\
\hline Colour (Pt.Co) & 15.0 & 5 \\
\hline $\mathrm{pH}$ & $6.5-8.5$ & $6.5-8.5$ \\
\hline $\mathrm{DO}(\mathrm{mg} / \mathrm{l})$ & 7.5 & \\
\hline $\mathrm{BOD}_{5}(\mathrm{mg} / \mathrm{l})$ & 0 & \\
\hline $\mathrm{COD}(\mathrm{mg} / \mathrm{l})$ & 0 & \\
\hline $\mathrm{Cl}(\mathrm{mg} / \mathrm{l})$ & 250 & $200-600$ \\
\hline $\mathrm{NO}_{2}(\mathrm{mg} / \mathrm{l})$ & 1.0 & 10 \\
\hline $\mathrm{NO}_{3}(\mathrm{mg} / \mathrm{l})$ & 10 & 50 \\
\hline $\mathrm{NH}_{4} \mathrm{~N}(\mathrm{mg} / \mathrm{l})$ & $<1.0$ & \\
\hline $\mathrm{SO}_{4}(\mathrm{mg} / \mathrm{l})$ & $200-400$ & 200 \\
\hline $\mathrm{P}(\mathrm{mg} / \mathrm{l})$ & $<5$ & \\
\hline $\mathrm{Na}(\mathrm{mg} / \mathrm{l})$ & 200 & \\
\hline $\mathrm{Mg}(\mathrm{mg} / \mathrm{l})$ & & 0.1 \\
\hline $\mathrm{Fe}(\mathrm{mg} / \mathrm{l})$ & 1 & 0.3 \\
\hline $\mathrm{Mn}(\mathrm{mg} / \mathrm{l})$ & $0.05-0.5$ & \\
\hline $\mathrm{Cu}(\mathrm{mg} / \mathrm{l})$ & 0.1 & $0.05-1.5$ \\
\hline $\mathrm{Zn}(\mathrm{mg} / \mathrm{l})$ & 5 & 5.0 \\
\hline $\mathrm{Pb}(\mathrm{mg} / \mathrm{l})$ & 0.05 & 0.01 \\
\hline $\mathrm{Cd}(\mathrm{mg} / \mathrm{l})$ & 0.01 & \\
\hline $\mathrm{Cr}(\mathrm{mg} / \mathrm{l})$ & 0.05 & \\
\hline $\mathrm{Ni}(\mathrm{mg} / \mathrm{l})$ & 0.05 & \\
\hline $\mathrm{V}(\mathrm{mg} / \mathrm{l})$ & 0.01 & \\
\hline
\end{tabular}

The TDS range obtained in this study is within the range reported by Sajitha and Vijayamma (2016) according to the researchers, Pond with low total dissolved solids points to the fact that, the water is less mineralized and has lesser pollutants comparatively. The TDS value reported by Shrivastava and Kanungo (2013) for Pond water of Surguja District, Chhattishgarh is higher than for this study, yet, within recommended range. Total Suspended Solids in water is undesirable for they decrease water transparency, inhibit photosynthesis, increase of bottom sediments, smother aquatic organism breeding bed (Ogbeibu and Anagboso, 2003/04). In pollution studies and waste treatment, total suspended solid is use to determine the strength of domestic wastewaters and to evaluate the efficiency of water treatment process (Rodajevic and Bashkin, 1999). Total dissolved solids of a water body indicate principally the different kinds of minerals in the water. The permissible value of TDS is $500 \mathrm{mg} / \mathrm{l}$.

The $\mathrm{pH}$ of the study area was generally acidic and within the range recorded for the then Okomu Forest Reserve by Jones (1955), Ogbeibu and Victor (1995). Low $\mathrm{pH}$ may be due to the massive growth of aquatic macrophytes shading the underlying water, reduced photosynthetic activity by phytoplankton and submerged macrophytes, and increased carbon dioxide level (Ogbeibu and Victor, 1995). They further observed that, the unstable phase of the ponds due to rapid water gain was characterized by increasing $\mathrm{pH}$ values, a trend which was reversed during the period of instability due to rapid water loss. Values of $\mathrm{pH}$ have been found to decrease with the growth of aquatic macrophytes (Kikuchi et al., 1976). Also, comparable $\mathrm{pH}$ range has been reported by Chindah and Braide (2001) for Bonny Brackish wetland and Ezekiel et al. (2011) in Sombreiro River. pH fluctuated between 5.90 (slightly acidic) - 7.35 (slightly alkaline) in Ponds of Agbede flood plain (Olomukoro et al., 2013). Abowei and George (2009) recorded pH of the Okpoka Creek, Niger Delta to be between 6.68 and 7.03 with minimal spatial variations this is in contrast within the findings of the present study where $\mathrm{pH}$ showed significant spatial variation. The acidic nature of African waters has been acknowledged by many researchers (Ajayi and Osibanjo, 1981; Iwegbue et al., 2007). Aquatic organisms are affected by $\mathrm{pH}$ because most of their metabolic activities are $\mathrm{pH}$ dependent (Wang et al., 2002). According to Adeyemo et al. (2008), problem associated with acidification is the solubilisation of some metals, when $\mathrm{pH}$ falls below 4.5. The hydrogen ion activity $(\mathrm{pH})$ of a solution is expressed as the logarithm of the reciprocal of the hydrogen ion activity at a particular temperature (Sajitha and Vijayamma, 2016). The acceptable limit of $\mathrm{pH}$ in drinking water by the WHO and Federal Ministry of Environment is between 6.5 - 8.5. 
Electrical conductivity is valuable in assessing the purity of water (Acharya et al., 2008). Sources of electrical conductivity include runoffs (Sajitha and Vijayamma, 2016). Electrical conductivity recorded during the present study in the Okomu River was between the range of $40-307.4 \mu \mathrm{s} / \mathrm{cm}$ whereas, a range of $12-21 \mu \mathrm{s} / \mathrm{cm}$ was recorded by Ogbeibu and Victor (1995) for the same River. The concentrations of electrical conductivity in the present study were less than the range of 14$41000 \mu \mathrm{s} / \mathrm{cm}$ recorded for New Calabar River (Ekeh and Sikosi, 2003) but within the range of 6$643 \mu \mathrm{s} / \mathrm{cm}$ recorded for other studies (Ogbeibu and Ezeunara, 2002; Sajitha and Vijayamma, 2016) but slightly less than range for pond water quality in Surguja District, Chhattisgarh (Shrivastava and Kanungo, 2013). The higher mean electrical conductivity values in Pond than River and Stream of the study area could have been influenced by the presence of organic matter and the concentration of ions during drying up of the ponds when there is little or no rain. Sikoki and Veen (2004) observed a conductivity range of $3.8-10 \mu \mathrm{s} / \mathrm{cm}$ in Shiroro Lake (Imo State) which was described as extremely poor in chemical composition. The limit of EC in drinking water is $1.500 \mu \mathrm{s} / \mathrm{cm}$ (Sajitha and Vijayamma, 2016). Though salinity for the Okomu Wetland varied significantly spatially, it indicated that the source is freshwater.

Ogbeibu and Victor (1995) reported a range of $0-9.7 \mathrm{mg} / \mathrm{l}$ DO levels. They attributed low levels of dissolved oxygen in ponds to the 'dense vegetation cover affecting oxygen diffusion and the high oxygen demand of microorganisms during oxidation of dead drowned macrophytes and other organic debris, facilitated by the reduced pond volume and increased temperatures. Dissolved oxygen (DO) is an important indicator of water quality, ecological status, productivity and health of a river (Moshood, 2008). In the present study, dissolved oxygen was within the range of $4.12-5.81 \mathrm{mg} / \mathrm{l}$ reported by Davies (2009) for the Woji-Okpoka Creek. Idowu and Ugwumba (2005) recorded dissolved oxygen concentrations ranging from $6.3-8.3 \mathrm{mg} / \mathrm{l}$. The authors attributed the high concentrations of dissolved oxygen to low organic enrichment. Arimoro et al. (2008) reported the range of 1.4 to $6.7 \mathrm{mg} / \mathrm{l}$ for the Warri River. The permissible value recommended by The Federal Ministry of Environment, Nigeria for DO is $5 \mathrm{mg} / \mathrm{l}$. Low dissolved oxygen has been reported for Charles Harbour, South Carolin (Lerberge et al., 2000). Low dissolved oxygen content and high biochemical oxygen demand is owed to high level of organic matter undergoing decomposition (Ogbeibu and Ezeunara, 2002). DO levels below $5 \mathrm{mg} / \mathrm{L}$ may negatively impact survival of biological populations within a water column and DO below 2mg/L may cause death in many fish species (Chapman and Kimstach 1996). The biological oxygen demand of a water body is the indication of organic wastes decomposable by bacteria. The BOD of the study area for all studied stations and water types was above the zero limit recommended by the Federal Ministry of Environment. Klein (1972) in River classification reported waters of medium to good quality and unpolluted as having biological oxygen demand below $6 \mathrm{mg} / \mathrm{l}$. Getachew et al. (2012) attributed low levels of DO and high BOD in their studies to anthropogenic activities such as intensive agricultural practices and illegal disposal of waste materials into the wetlands. Chemical oxygen demand (COD) is the amount of the oxygen which is equivalent to the portion of the organic matter susceptible to oxidation in a test condition (Bertram and Balance, 1996). The levels of COD in the present study suggests a greater amount of oxidizable organic materials especially in the ponds of the study area, and this could have led to a reduction in the amount of dissolved oxygen present.

Egborge (1971) opined that the growth and metabolism of phytoplankton are partly responsible for low levels of dissolved plant nutrients including nitrates in waters. The leaching of plants and dry leaf litter contribute nitrate to water bodies (Hynes, 1970; Wetzel, 1974). In this study, the variations of phosphate levels reflected spatial variations. The mean concentrations of ammonium- $\mathrm{N}$ and nitrate for this study were higher than that recorded by Chindah and Braide (2001) at Bonny Brackish Wetland of the Niger Delta. The WHO's permissible limit of nitrate is $50 \mathrm{mg} / \mathrm{l}$. The recorded mean concentrations of available phosphorous compared favorably with the mean values obtained by Nnaji et al. (2011) at River Galma, Zaria. Excess phosphorous in water is considered a pollutant (Odiete, 1999). Recommended limit is not available for sulphate in drinking water because it is said to be one of the least toxic anions, yet, dehydration, gastrointestinal irritation and catharsis, have been linked to high concentrations of sulphate in drinking water. The World Health Organisation therefore advises an urgent action be taken by health authorities when sulphate in drinking water exceeds $500 \mathrm{mg} / \mathrm{l}$ (WHO, 1992; 1999). In the present study, the sulphate values were below 500mg/l. 
Calcium concentrations were generally higher in the temporary ponds than in Okomu River and the Arhakhuan Stream and significant variations were observed. These observed values fall within the findings of Omoigberale and Ogbeibu (2007) for Osse River Southern Nigeria (1.1 to $9.62 \mathrm{mg} / \mathrm{l}$ ). Calcium is often the most common metallic ion in fresh surface water and among the most common in ground water. Sources of calcium in fresh water are the bed rock and weathering of calcium bearing rocks (Waite, 1984). The concentration of magnesium is generally lower than calcium even though it is often associated with calcium in all kinds of water (Venkatasubramani and Meenambal, 2007). The level of magnesium recorded in this study showed significant spatial variations and low when compared to the levels of River Oshun which ranged between 1.0 - 5.6mgl (Egborge, 1971), (0.2 $7.78 \mathrm{mgl}$ ) for Osse River (Benin axis) reported by Omoigberale and Ogbeibu (2007), and River Jamieson (0.1 - 72.9mgl) (Imoobe and Oboh, 2003). The concentration of Magnesium was generally higher in the ponds than in River and Stream respectively. The distribution of calcium and magnesium clearly indicate that all the samples lie within the desirable limit $(75 \mathrm{mg} / \mathrm{l}$ and $30 \mathrm{mg} / \mathrm{l}$ respectively) for drinking water (WHO, 1999). Sodium is a natural component of water, but its concentration can be increased by pollution and high concentrations of $\mathrm{Na}$ gives bitter taste. These sources of pollution of $\mathrm{Na}$ include rock salt, precipitation and runoff (Sajitha and Vijayamma, 2016). The concentration of sodium in the present study showed significant spatial variations. The sodium values obtained were low when compared with the levels in River Sokoto 3.1-11.0mg/l (Holden and Green, 1960). Sodium concentration of more than $50 \mathrm{mg} / \mathrm{l}$ makes water unsuitable for drinking (WHO, 1999). The potassium level of the water bodies within the study area were low when compared with the level in River Sokoto (2.8 to $13.2 \mathrm{mg} / \mathrm{l})$ as reported by Holden and Green (1960). The cations in water bodies of the study area are in the order $\mathrm{Ca}>\mathrm{Mg}>\mathrm{Na}>\mathrm{K}$.

Heavy metals concentrations of the Wetland were generally low. Low heavy metal concentrations have been reported in Nigerian water bodies by earlier workers, notable among are Obire et al. (2003) on Elechi Creek, Chindah and Braide (2004) on lower Bonny River, Omoigberale and Ogbeibu (2005) on the Osse River, Southern Nigeria. The high toxicity of heavy metals to aquatic life and humans make them important indices of pollution (Omoigberale and Ogbeibu, 2005). Heavy metals show significant variation but were within the recommended standards by the World Health Organization.

\subsection{Conclusion}

The Okomu Wetland harbors water bodies which include temporary/seasonal ponds, the Arhakhuan Stream and the Okomu River. All physicochemical parameters investigated varied significantly and principally influenced by the nature of the water bodies, water level, topography, forest canopy cover, surface run-off and time of sampling. This paper documented the present status of the physicochemical conditions of the Okomu Wetland, providing a benchmark for future comparative investigations.

\section{Acknowledgements}

We acknowledge the support of Management and Staff of; Okomu National Park, Martlet Laboratories, Tobejay Technologies, SPDC (Shell Petroleum Development Company) Nigeria - Post Graduate Internship Programme, and JEFFA Geosurveys and Technical Services Limited during the course of this research work. Special thanks to Mike Oiwoh and Cyril Ukpamufo Olowo (amongst others) for their assistance in some aspects of field data gathering.

\section{References}

Abowei, J. F. N and George, A. D. I. (2009). Some physical and chemical characteristics in Okpoka creek, Niger Delta, Nigeria. Res. J. Environ. Earth Sci. 1, pp. 45-53.

Acharya, G. D., Hathi, M. V., Patel, A. D. and Parmar, K. C. (2008). Chemical properties of groundwater in Bhiloda Taluka Region, North Gujarat. India. E-Journal of Chemistry, 5(4), pp. 792796.

Adebisi, A. A. (1981). The physicochemical hydrology of a tropical seasonal river upper Ugon River. Hydrobiology. 79, pp. $157-165$. 
Adeyemo, O. K., Adedokun, O. A., Yusuf, R. K. and Adeleye, E. A. (2008). Seasonal changes in physicochemical parameters and nutrient load of River sediments in Ibadan City, Nigeria. Global NEST Journal, 10(3), pp. 326 - 336.

Ajayi, S. S. (2001). Multi-purpose forest management for bush meet production: A success story from West Africa. http://www.fao.org. Accessed on 11/03/2014.

Ajayi, S. O. and Osibanjo, O. (1981). Pollution studies of Nigerian rivers, II water quality of some Nigerian Rivers. Environ. Pollut. 2(8), pp. 87-95.

Akpan, E. R., Ekpo, H. E. and Ekpe, U. J. (2003). Seasonal variation in water quality of the Calabar River: influence of tidal and coastal activities. Global Journal of Environmental Sciences, 2(2), pp. 106-110.

American Public Health Association /American Water Works Association/Water Pollution Control Federation (1998). Standard methods for the examination of water and waste water. 20th Ed. APHA, Washington, DC 1325pp.

Anadu, P. A. and Oates, J. F. (1982). The status of wildlife in Bendel State, Nigeria. With recommendations for its conservation. Mimeo report to the Nigerian Government, the New York Zoological Society and the World Wildlife Fund, (U.S.)

APHA (2005) Standard Methods for the Examination of water and wastewater. American Public Health Association, Washington D. C., pp. 1000.

Arimoro, F. O., Iwegbue, C. M. A. and Osiobe, O. (2008). Effects of Industrial Waste Water on the Physical and Chemical Characteristics of a Tropical Coastal River. Research Journal of Environmental Sciences, 2, pp. 209-220.

Asibor, G. (2009). Wetlands: values, uses and challenges. A Paper presented to the Nigerian Environmental Society at the Petroleum Training Institute, Effurun, $21^{\text {st }}$ November, 2009.

Asonye, C. C., Okolie, N. P., Okenwa, E. E. and Iwuanyanwu, U. G. (2007). Physiochemical characteristics and heavy metal profiles of Nigerian rivers, streams and waterways. African journal of Biotechnology, 6 (5), pp. 617-624.

Bamgboye, O. A., Osundiya, M. O., Olowu R. A. and Adeniyi, D. A. (2016). Physicochemical Analysis of Wetland Water of Iba Community in Lagos State Nigeria. American Journal of Chemistry, 6(6) pp. 149-154.

Barbier, E. B., Acreman, M. and Knowler, D. (1997). Economic Valuation of Wetlands, A Guide for Policy Makers and Planners Ramsar Convention Bureau, Gland, Switzerland.

Bertram, J. and Balance, R. (1996). "A Practical Guide to the Design and Implementation of Freshwater, Quality Studies and Monitoring Programmes". United Nations Environmental Programmes (UNEP) and World Health Organization (WHO). E and F.N Spoon Publishers.pp.172177.

Chapman, D. and Kimstach, V (1996). Selection of water quality variables. Edited by Deborah Chapman. Water Quality Assessments - A Guide to Use of Biota, Sediments and Water in Environmental Monitoring - Second Edition. UNESCO/WHO/UNEP.

Chindah, A. C. and Braide, S. A. (2001). Meiofauna occurrence and distribution in different substrate types of Bonny Brackish wetland of the Niger Delta. J. Appl. Sci. Environ. Mgt. 5(1), pp. 33-41.

Chindah, A. C. and Braide, S. A. (2004). The physicochemical quality and phytoplankton community of tropical waters: A case of 4 biotopes in the lower bonny river, Niger Delta, Nigeria. Caderno de Pesquisa Serie Biol., 16, pp. 7-35. 
Davies, O. A. (2009). Spatio-temporal Distribution, Abundance and Species Composition of zooplankton of Woji-okpoka Creek, Port Harcourt, Nigeria. Res. J. Appl. Sci. Eng.Technol., 1(2), pp. 14-34.

Egborge, A. B. M. (1971). The Chemical Hydrology of the River Oshun, Western State, Nigeria. Fresh Water Biology, 1(3), pp. 257 - 272.

Ekeh, I. B. and Sikoki, F. D. (2003). The state and seasonal variability of some physicochemical parameters in the New Calabar River, Nigeria. Supp. Ad Acta Hydrobiol., 5, pp. 45-60.

Emelue, G. U. and Ukandu, M. (2014). Perception of host communities toward the implementation of park laws in Okomu National Park Jornal of Research in Forestry, Wildlife and Environment. 6 (2), pp. $90-98$.

Ezekiel, E. N., Hart, A. I. and Abowei, J. F. N. (2011). The Sediment, Physical and Chemical Characteristics in Sombreio Rivers, Niger Delta, Nigeria. Res. J. Environ. Earth Sci., 3(4), pp. 43-349.

Getachew, M. Ambelu, A. Tiku, S. Legesse, W. Adugna, A. and Kloos, H (2012). Ecological assessment of Cheffa Wetland in the Borkena Valley, northeast Ethiopia: Macroinvertebrate and bird communities. Ecological Indicators, 15, pp. 63-71.

Holden, M. J. and Green J. E. (1960). The hydrology and plankton of the River Sokoto. J. Animal Ecology 29(1), pp. 65-84.

Hynes, H. B. N. (1970). The ecology of running waters. Liverpool Univ. Press, Liverpool.

Idowu, R. T. and Ugwumba, A. A. (2005). Physical, chemical and benthic characteristics of a southern Nigeria Reservoir. The zoologist, 3, pp. 15-28.

Ikusima, I., Lim, R. P. and Furtado, J. I. (1982). Environmental conditions in: J.I. Futado and S. Mori (eds). Tasek Bera the Ecology of a Tropical Freshwater swamp. Dr. W. Junk, The Hague. pp.55-148.

Imoobe, T .O. T. and Oboh, I. P. (2003). Physical and chemical hydrology of river Jamieson, Delta State, Nigeria. Benin Sci Digest., 1, pp. 105-119.

Iwegbue, C. M. A., Nwajei, E. G. and Arimoro, F. O. (2007). Assessment of contamination by heavy metals in the sediment of Ase River, Niger Delta, Nigeria. Research Journal of Environment Science, $1(5)$, pp. 220-228.

Jones, E. W. (1955). Ecological studies on the rainforest of southern Nigeria. IV. The Plateau Forest of the Okomu Forest Reserve. J. Ecol., 44, pp. 564-594.

Kikuchi, E., Furusaka, C. and Kurihara, Y. (1976). Surveys of the fauna and flora in the water and soil of paddy fields. The reports of the Inst. For Agric. Res. Tohoku Univ., 26, pp. 25 - 35.

Klein, L. (1972). River; pollution control, Butterworths and Company, London, 13th (Ed.).

Lerberge, S. B., Holland, A. F. and Sanger, D. M. (2000). Response of tidal creek macrobenthic communities to the effects of water shed development. Estuaries, 23 (6), pp. 838-853.

Matthews, G. V. T. (1993). The Ramsar Convention on Wetlands: its history and development. In: Luthi E, Ramsar, editors. Gland (Switzerland): Ramsar Convention Bureau.

Millenium Ecosystem Assesment (2005). Ecosystems and Human WellBeing: Wetlands and Water Synthesis. World Recourses Institute, Washington, DC.

Moshood, K. M. (2008). Assessment of the water quality of Oyun reservoir, Offa, Nigeria, using selected physicochemical parameters. Turkish Journal of Fisheries and Aquatic Sciences 8: 309-319. 
Nigeria National Park Service: Okomu National Park. Retrieved from http://nigeriaparkservice.org/okomu /Default.aspx on 21st of April, 2015.

Nnaji, J. C., Uzairu, A., Harrison, G. F. S. and Balarabe, M. L. (2011). Effect of pollution on the physicochemical parameters of water and sediments of river Galma, Zaria, Nigeria. Research journal of environmental and earth sciences, 3(4), pp. 314-320.

Obire, O., Tamuno, D. C. and Wemedo, S. A. (2003). Physicochemical quality of Elechi Creek in Port Harcourt, Nigeria. J. Appl. Sci. Environ. Manage. 7, pp.43-49.

Odiete, W. O. (1999). Environmental physiology of animals and pollution. Diversified Resources, Lagos, Nigeria, pp. 220-246.

Ogbeibu A. E and Victor R. (1995). Hyrobiological studies of water bodies in the Okomu Forest Reserve (Santuary) in Southern Nigeria. 2: physical and chemical hydrology. Tropical freshwater Biology, 4, pp. 83-100.

Ogbeibu A. E. and Egborge A. B. M. (1995). Hydrobiological studies of water bodies in the Okomu Forest Reserve (Sanctuary) In Southern Nigeria. 1. Distribution and Diversity of the invertebrate fauna. Tropical Freshwater Biology, (4), pp. 1-27.

Ogbeibu, A. E. and Anagboso, M. U. (2003/04). Baseline limnological investigation of the Utor River in Esan southeast, Edo state, southern Nigeria: Physical and chemical hydrology. Tropical freshwater biology, 12/13, pp. 45-62.

Ogbeibu, A. E. and Edutie, L. O. (2002). Impact of Brewery Effluent on the Water Quality and Rotifers of Ikpoba River, Southern Nigeria. African Journal of Environmental Pollution and Health, $1(1), \mathrm{pp} 1-12$.

Ogbeibu, A. E. and Ezeunara, P. U. (2002). Ecological Impact of Brewery Effluent on the Ikpoba River, Using the Fish Communities as Bioindicators. Journal of Aquatic Sciences, 17(1), pp. 35 - 44.

Okonkwo, C. N. P., Kumar, L. and Taylor, S. (2015). The Niger Delta wetland ecosystem: What threatens it and why should we protect it? Afr. J. Environ. Sci. Technol., 9(5), pp. 451- 463.

Olaleru, F. and Egonmwan, R. I. (2014). Wildlife Conservation Challenges in Okomu National Park, Nigeria. Ethiopian Journal of Environmental Studies and Management, 7(6): 670 - 676.

Olomukoro, J. O., Idiaghe M. O. and Abdul-Rahman, D. (2013). Ecological Survey of Macrobenthic Invertebrates of Selected Ponds in Agbede Flood Plain, Southern Nigeria. Journal of Biology, Agriculture and Healthcare. 3(10), pp. 23-29.

Omoigberale, M. O. and Ogbeibu, A. E. (2005). Assessing the environmental impacts of oil exploration and production on the Osse river, Southern Nigeria, I. Heavy metals. African Journal of Environmental Pollution and Health, 4(1), pp. 27-32.

Omoigberale, M. O. and Ogbeibu, A. E. (2007). Environmental impacts of oil exploration on the Crustacean Zooplankton of Osse River, Southern Nigeria. J. Sci. Ind. Res., 50, pp. 266-272.

Radojevic, M. and Bashkin, V. N. (1999). Practical Environmental Analysis. Royal Society of Chemistry, Cambridge, pp. 466.

Ramsar Convention Secretariat (RCS) (2007) Wise use of wetlands: a conceptual framework for the wise use of wetlands Ramsar handbooks for the wise use of wetlands. 3rd edn. Ramsar Convention Secretariat Gland, Switzerland.

Sajitha, V. and Vijayamma, S. A. (2016). Study of Physicochemical Parameters and Pond Water Quality Assessment by using Water Quality Index at Athiyannoor Panchayath, Kerala, India. Emer 
Life Sci Res., 2(1), pp. 46-51.

Shrivastava, S. Kanungo, V. K. (2013). Physicochemical Analysis of Pond Water of Surguja District, Chhattishgarh, India. International Journal of Herbal Medicine, 1(4), pp. 35-43.

Sikoki, F. D. and Veen, J. V. (2004). Aspects of water quality and potential for fish production of Shiroro Reservior, Nigeria. Livestock Syst. Sustainable Dev. 2, pp. 1-7.

Turner, R. K., van den Bergh J. C. J. M., Söderqvist T., Barendregt, A., van der Straaten, J., Maltby, E., van Ierland, E. C. (2000). Ecological-economic analysis of wetlands: scientific integration for management and policy. Ecol Econ., 35, pp.7-23

Uluocha, N. and Okeke, I. (2004). Implications of wetlands degradation for water resources management: lessons from Nigeria. Geo. J., 61:151

UNESCO/WHO/UNPP, (1992). Water Quality Assessment. A Guide to use of Biota, Sediments and Water in Environment Monitoring. 2nd Edn. pp. 306.

Venkatasubramani, R. and Meenambal, T. (2007). study of subsurface water quality in mattupalayam Taluk of Coimbatore district Tamil Nadu. Nat. Environ. Poll. Tech., 6, pp. 307-310.

Waite, T. D. (1984). Principle of water quality. Academic Press. San Diego.

Wang, W., Wang, A., Chen, L., Liu Y. and Sun, R. (2002). Effects of pH on Survival, Phosphorus.

Wetzel, R. G. (1974). Review of: A Guide to the Measurement of Marine Primary Production under some Special Conditions. (UNESCO, Paris, 1973). Trans. Amer. Fish Soc., 103, pp. 416-417.

Cite this article as:

Ogbeibu A. E. and Ehiorobo S. I., 2020. Physicochemical Characteristics of the Okomu Wetland, Edo State, Nigeria. Nigerian Journal of Environmental Sciences and Technology, 4(2), pp. 398-410. https://doi.org/10.36263/nijest.2020.02.0225 